\title{
The Long-Term Behaviour of BY Dra
}

\author{
K.P. Panov ${ }^{1}$, M.S. Ivanova ${ }^{1}$, J.S.W. Stegert ${ }^{2}$ \\ 1 Institute of Astronomy, Bulgarian Academy of Sciences, Zarigradsko Shose 72, \\ 1748 Sofia, Bulgaria \\ 2 Sternwarte der Universität Bonn, Auf dem Hügel 71, 53121 Bonn, Germany
}

BY Dra has been extensively monitored for flares and spot-modulation of brightness at the Bulgarian National Astronomical Observatory (Panov \& Ivanova 1993). Its mean light level exhibited remarkable changes in the past, due to the emergence and disintegration of photospheric spots, and different cycles have been proposed for the long-term changes of the BY Dra activity. This star is still believed to be a good candidate for cyclic variations, presumably similar to the sunspot activity cycle. After a spectacular drop in brightness in 1965-1967, and a subsequent rise in the early $70 \mathrm{~s}$, the mean light remained approximately constant during 1977 - 1991. A brightening of BY Dra occurred in 1992.

In Fig. 1, the light curves are shown for 1991,1992 and 1993 in the $V$-band and in $B-V$. Phases were calculated with the ephemeris of Chugainov (1966):

$$
\text { JD }(\text { min. light })=2438983.612+3.836 \cdot E
$$

From Fig. 1, it is apparent that the mean visual light, which was about $V=8.20$ in 1991 , increased in 1992 by $00^{\mathrm{m}} 10$, and a further increase by another $0^{\mathrm{m}} 03$ occurred in 1993. The phase of minimum light in 1992 indicates that the position of the spot remained approximately the same in stellar longitude as in 1991 . However, in 1993 the position of the spot changed by almost half a period.

In Fig. 2, the phase of minimum light is plotted versus JD for $1986-1992$. The smooth variation indicates that the light modulation during that time was dominated by the same spot (or spot-group), which migrated either in stellar longitude, or in latitude. In the second case, the phase variations reflect the rotation on different stellar latitudes, i.e. differential rotation. This spot disintegrated in 1992, resulting in an increase of the mean light. The spot phase of 1993 indicates that probably a new spot emerged on the opposite side of the star.

In the 1992 light curve (Fig. 1), the deviation of the encircled point is probably due to the rapid shift of the light curve in ordinate during the observing season. It reflects a brightening of $0^{\mathrm{m}} 06$ from JD 2448780.5 to 2448835.4 . The $B-V$ curves have an amplitude of about $0^{\mathrm{m}} 015$ in both 1992 and 1993, and the colour is reddest at light minimum. This is in agreement with a dark-spot model. It is interesting to note, that the $0{ }^{\mathrm{m}} 13$ brightening of BY Dra resulted in a blue shift of only 0.01 in $B-V$.

In Fig. 3, the long-term behaviour of the BY Dra is shown in the $V$ band and in $B-V$. The 1993 , the mean visual light is about the same as in the early 
1991

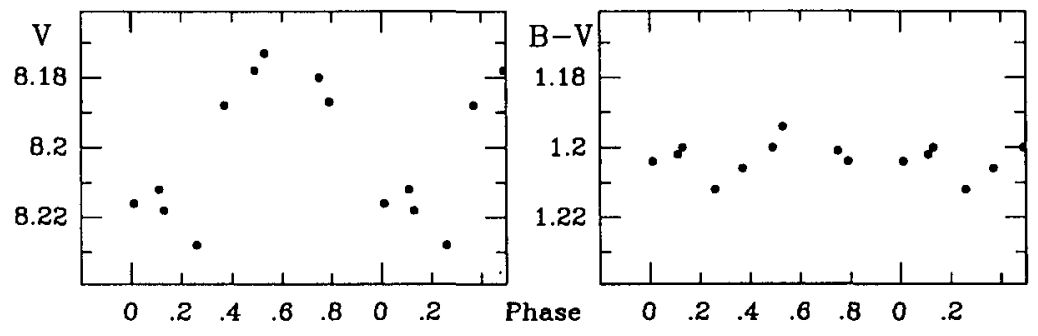

1992

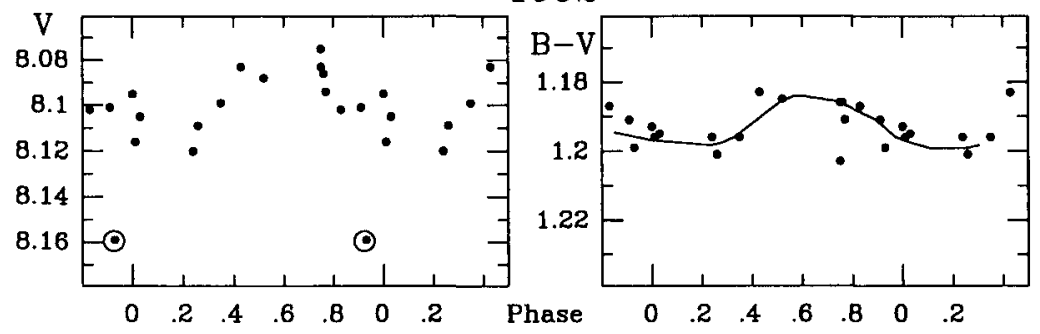

1993

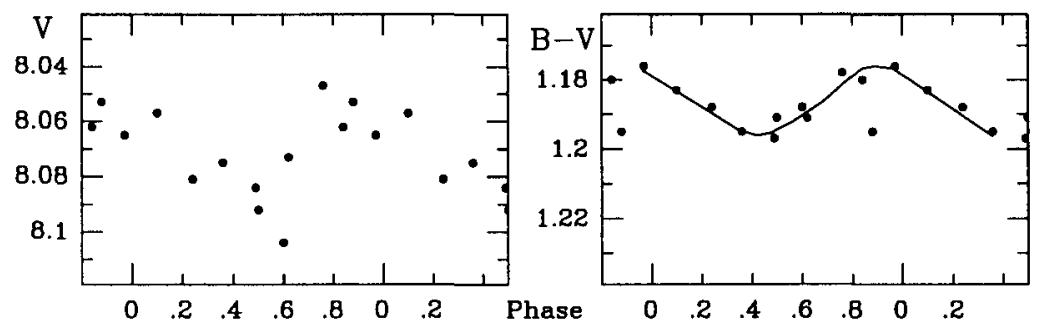

Fig. 1. Light curves of BY Dra in 1991, 1992 and 1993. Data from the Bulgarian National Observatory.

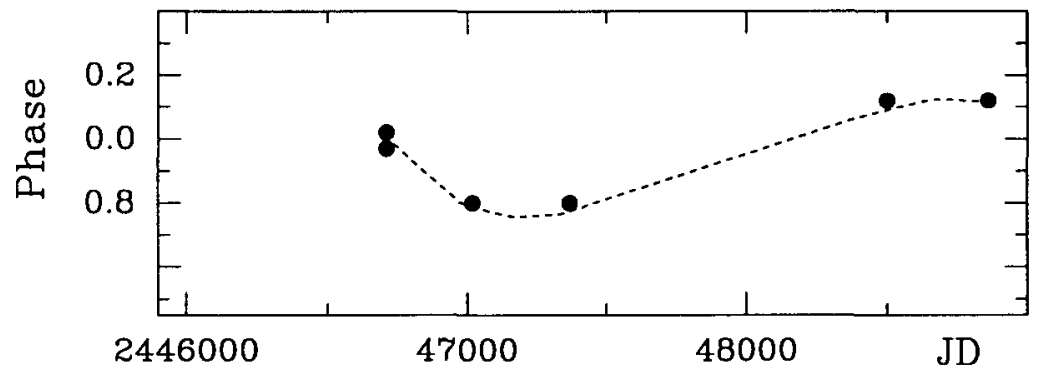

Fig. 2. Phase of minimum light of BY Dra versus JD for $1986-1991$. 


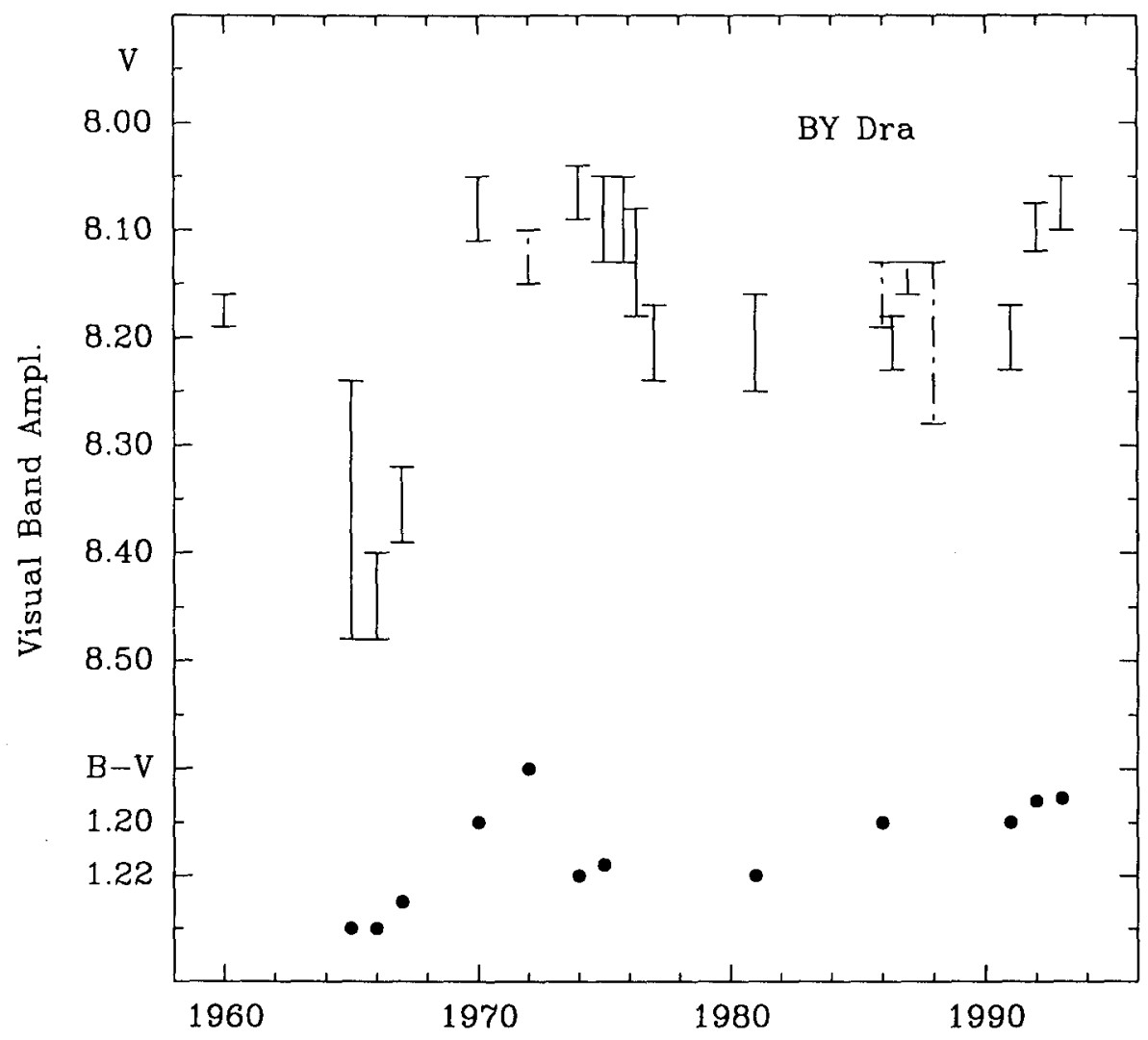

Fig. 3. The long-term mean light variations of BY Dra (from published data and the present work)

70s. The complicated character of the long-term variations does not confirm previously proposed activity cycles for BY Dra, and any suggestion for a cycle length seems as yet premature.

Acknowledgements: This work has been supported by the Bulgarian National Science Foundation with grant F-465 and by the Deutsche Forschungsgemeinschaft (Projekt 436 BUL 113).

\section{References}

Chugainov P.F., 1966, IBVS No. 122

Panov K.P., Ivanova M.S., 1993, ApSS 199, 265 